\title{
The Power Output and Capacity Configuration Method of Hybrid Energy Storage Systems Considering the Confidence Level of PV Fluctuation
}

\author{
Jin Zong, Kai Bai, Bo Yuan, and Zhi Li
}

\begin{abstract}
The fluctuation of Photovoltaic (PV) generation brings significant impact on power systems. To reduce such impact, this paper proposed a power and capacity configuration method for hybrid energy storage systems (EES) considering the confidence level of PV output fluctuation. The spectral analysis and lowpass filtering (LPF) methods are combined, and according to the spectral analysis results of PV output, the optimized second-order LPF time constant is determined by considering the confidence level of fluctuation rate, integrated power fluctuation constraints, charging and discharging power balance constraints, charging-discharging efficiency of energy storage equipment and the state of charge. Then the output of hybrid PV-ESS and the charging and discharging power and capacity of each components of the hybrid energy storage system that satisfies the power fluctuation demand can be calculated. Simulation is carried out using Matlab to validate the effectiveness of the algorithm on smoothing PV output fluctuation.
\end{abstract}

Index Terms-Hybrid energy storage, fluctuation rate, spectral analysis, lowpass filtering, charging and discharging power balance.

\section{INTRODUCTION}

With ever increasing PV penetration, the intermittent and random characteristic of Photovoltaic (PV) has brought significant impact on the stable and reliable operation of power systems [1]. Due to the rapid adjustment ability of power electronic devices, energy storage system (EES) has become an effective way to deal with the random fluctuation of PV power [2]. The hybrid EES consists of super capacitor and storage battery. It can make full use of the two kinds of accumulators and improve the operating condition of storage battery, therefore to extend its lifespan [3]-[5].

Currently, the main EES capacity configuration method to smooth new energy generation output is to establish a quantitative model with the objective of minimal energy

Manuscript received October 15, 2015; revised March 9, 2016. This paper is funded by the National High Technology Research and Development of China (863 Programme: 2011AA05A113) and Wind-Solar-Energy Storage Hybrid Power Generation Technology Laboratory.

Jin Zong, Kai Bai, and Zhi Li are with State Grid Jibei Electric Power Co., Ltd Research Institute, China (e-mail: hyacinthzj@126.com, baikai1234@263.net, lizhi18688575@126.com).

Bo Yuan is with State Grid Energy Research Institute, China (e-mail: yuanbo@sgeri.sgcc.com.cn). storage device capacity or minimal cost [6]. Then according to certain constraints, configuration model and algorithm to solve the model and achieve the optimal capacity of EES. However, there has been few such model that can be used in actual project. In [7], filter is deployed to dispatch of power output of EES. The high-frequency component is compensated by power type energy storage, and the low-frequency component is compensated by energy type energy storage. Then the charging and discharging instructions of the hybrid EES can be obtained. However, the method to determine filtering constant and capacity optimization method is not given. In [8], the rain-flow calculation method is used to calculate the battery discharge depth, and the battery life quantification model is built by equivalent life circle curve; it has proved that replacing single-type battery energy storage system with hybrid energy storage system can greatly reduce operation cost. But the control strategy doesn't involve the real time operation status and charging-discharging balance. In [9], to minimize the initial construction cost of energy storage system is considered, and fuzzy control strategy is used to modify reference power value, and the capacity and output of Li-battery and supercapacitor is decided. But the SOC variation range of Li-battery is limited to be very small in this method, and the Li-battery capacity is relatively large, which can extend its life span, but may reduce economy.

Although there are a lot of research on hybrid EES currently, but most of them focused on capacity optimization. Such as in [10], a multi-objective optimization model is built from three aspects: peak shaving, voltage quality and active power adjustment. It has deployed particle swarm optimization (PSO) algorithm to solve the model. But the PSO cannot simulate the real operation status of the hybrid EES.

This paper proposed a capacity and output configuration method for hybrid EES considering the confidence level of PV output fluctuation rate. The spectral analysis and lowpass filtering (LPF) methods are combined, and according to the spectral analysis results of PV output, the optimized second-order LPF time constant is determined by considering the confidence level of fluctuation rate, integrated power fluctuation constraints, charging and discharging power balance constraints, charging-discharging efficiency of energy storage equipment and the state of charge. Then the output of hybrid PV-ESS and the charging and discharging power and capacity of each components of the hybrid energy storage system that satisfies the power fluctuation demand can be calculated. 


\section{PV OUTPUT CHARACTERISTIC}

\section{A. Selection of PV Data Sample}

The charge and discharge cycle of the supercapacitor of the hybrid EES is relatively short, approximately 10-30 seconds. According to sampling theorem and considering the current monitoring system, the sampling interval is set to be 5-15 seconds. As to the choosing of PV data sample, according to the output curve of different season and weather, the EES is used to smooth PV output fluctuation. The sample period is set to be one day.

\section{B. Statistics of PV Output Fluctuation Rate}

The national standard of grid-integrated PV output fluctuation rate in China is "the variation speed of PV real power should be less than $10 \%$ of the installed capacity per minutes" [11]. There is no such standard for distributed PV currently. The fluctuation rate limitation of PV output and its confidence level can be determined according to the operation characteristic of local distribution network and load characteristic. The fluctuation rate of PV output can be calculated by:

$$
F_{t}=\frac{\Delta P_{t}}{P_{\mathrm{n}}}=\frac{P_{t \max }-P_{t \min }}{P_{\mathrm{n}}} \times 100 \%
$$

where $P_{n}$ is the PV rated power; $P_{t}$ is the max power change during time period $t ; P_{\max }$ and $P_{\min }$ is the maximum and minimum output during time period $t$.

The confidence level of PV output fluctuation rate is determined according to statistical results. It is the percentage $\mathrm{P} \%$ of the time period that can meet the fluctuation rate demand after adding EES to the total time period.

$$
P \%=t_{1} / t_{\text {total }}
$$

where $t_{1}$ is the sample point when the sample can meet the fluctuation rate demand; $t_{\text {total }}$ is the number of all the sampling points.

\section{Spectral Analysis of PV Output}

Applying discrete Fourier transform to the sample data of PV output:

$$
P(k)=\sum_{n=0}^{N-1} P(n) \cdot e^{-j \frac{2 \pi}{N} k n}, \quad k=0,1, \ldots, N-1
$$

By analyzing it amplitude-frequency characteristic, we can get the major frequency range, where $\omega_{L}$ and $\omega_{H}$ are the upper limit and lower limit of fluctuation rate range. Then the filtering time constant $\left[T_{L}, T_{H}\right]$ can be calculated.

\section{MATH}

Appling second-order low-pass filtering (LPF) to PV output (see Fig. 1).

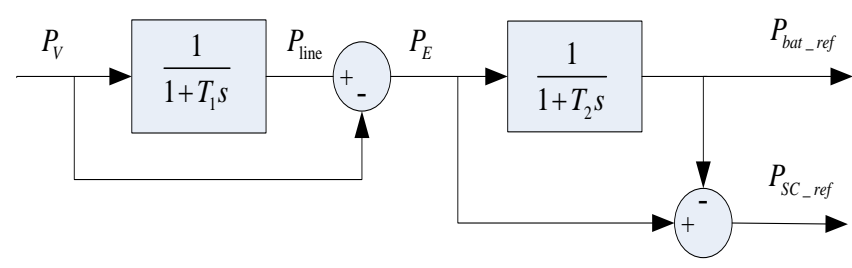

Fig. 1. Schematic diagram of second-order lowpass filtering.

$$
\begin{gathered}
P_{\text {line }}=\frac{1}{1+T_{1} s} P_{V} \\
P_{E}=-\frac{1}{1+T_{1} s} P_{V} \\
P_{\text {bat_ref }}=\frac{1}{1+T_{2} s} P_{E} \\
P_{S C_{-} \text {ref }}=\frac{T_{2} s}{1+T_{2} s} P_{E}
\end{gathered}
$$

where $P_{V}$ is the output of $\mathrm{PV} ; P_{\text {line }}$ is the grid tie line power; $P_{E}$ is the power of energy storage compensation; $P_{b a t \text { ref }}$ is the reference output power of Li-battery; $P_{S C_{-} r e f}$ is the reference output power of supercapacitor; $T_{1}$ is the first-order filtering time constant; $T_{2}$ is the second-order filtering time constant.

Choose a filtering time constant from small to large from $\left[T_{L}, T_{H}\right]$, and calculate the fluctuation rate and confidence level of the output of the PV-energy storage generation system until the desirable confidence level is achieved. This constant is the first-order filtering time constant. The second-order lowpass filtering is then carried out in the same way. The second-order filtering time constant is usually set to be 10-30 seconds when Li-battery and supercapacitor are used in hybrid ESS. The reference output value of Li-battery and supercapacitor is then obtained.

\section{CAlCUlation OF THE RATED POWER OF HYBRID ENERGY STORAGE}

The reference output value that need to be compensated should ensure the stable operation and consider the energy loss during the charging and discharging process of the hybrid ESS. The time period for Li-battery capacity calculation is usually set to be one day. The charging time of super capacitor is relatively short, therefore its calculation time period is usually set to be 1-5 minutes.

The adjustment is carried out as follows:

Step 1) Translation the power instructions of the super capacitor during each $T_{S C}$ until the sum of them is zero;

Step 2) Translation the power instructions of the Li-battery in opposite direction, and let the total power instructions of the hybrid ESS stay unchanged;

Step 3) Re-modify the modified power instructions of the Li-battery during $T_{\text {bat }}$, and let the sum be 0 . This could ensure the energy balance of the hybrid ESS. $T_{S C}, T_{\text {bat }}$ are the charging and discharging cycle of the super capacitor and the battery. 


$$
\begin{aligned}
& \Delta P_{S C}=\frac{1}{N} \sum_{1}^{N} P_{S C_{-} r e f}[n], n=1,2, \ldots N \\
& N=T_{S C} / T_{S} \\
& P_{S C}^{\prime}[n]=P_{S C}[n]-\Delta P_{S C}, n=1,2, \ldots N \\
& N=T_{S C} / T_{S} \\
& P_{\text {batx }}[n]=P_{\text {bat_ref }}[n]+\Delta P_{S C}, n=1,2, \ldots N \\
& N=T_{b a t} / T_{S} \\
& \Delta P_{b a t}=\frac{1}{N} \sum_{1}^{N} P_{b a t x}[n], \quad n=1,2, \ldots N \\
& N=T_{b a t} / T_{S} \\
& P_{b a t}^{\prime}[n]=P_{b a t x}[n]-\Delta P_{b a t}, \quad n=1,2, \ldots N \\
& N=T_{b a t} / T_{S} \\
& P_{\text {line }}{ }^{\prime}[n]=P_{\text {line }}[n]-\Delta P_{b a t}, n=1,2, \ldots N \\
& N=T_{b a t} / T_{S}
\end{aligned}
$$

where $\Delta P_{S C}$ and $\Delta P_{b a t}$ are the translation, $P_{S C}[n], P_{b a t}[n]$ and $P_{\text {line }}[n]$ are the power output of the super capacitor, Li-battery and connecting line after translation. $T_{S}$ is the sampling period.

During the whole period, the maximum value of the compensation power of each components of the hybrid ESS is the maximum charging and discharging power of each components, i.e. the rated power.

$$
P_{N}=\max \left\{\left|P^{\prime}[n]\right|\right\}
$$

\section{CALCUlation OF ESS RATED CAPACITY}

The calculation methods of Li-batter and super capacitor rated capacity are much the same. Take Li-battery as an example.

\section{A. Charging and Discharging Electric Quantity \\ Calculation}

After we obtain the output curve of the Li-battery, its charging and discharging electric quantity can be calculated as:

$$
E[n]=\sum_{1}^{n} P_{b a t}^{\prime}[i] \frac{T_{s}}{3600}, \quad n=1,2 \ldots N
$$

\section{B. Rated Capacity Calculation}

The real-time state of charge (SOC) of the ESS is:

$$
\begin{gathered}
E[n]=\sum_{1}^{n} P_{b a t}^{\prime}[i] \frac{T_{s}}{3600}, \quad n=1,2 \ldots N \\
S O C=S O C_{0}-\frac{E[n]}{E_{N}}
\end{gathered}
$$

$$
\left\{\begin{array}{l}
S O C_{0}-S O C_{\text {low }} \geq \frac{\max \{E[n]\}}{E_{N}} \\
S O C_{u p}-S O C_{0} \leq \frac{\min \{E[n]\}}{E_{N}}
\end{array}\right.
$$

Then we have

$$
E_{N}=\frac{\max \{E[n]\}+|\min \{E[n]\}|}{S O C_{u p}-S O C_{\text {low }}}
$$

where $S O C_{0}$ is the initial $S O C, E_{N}$ is the rated power of Li-battery; $S O C_{u p}$ and $S O C_{\text {low }}$ are the maximum and minimum value of SOC.

\section{CASE STUdy}

The test system is a $10 \mathrm{kV}$ distribution network with distributed PV integrated. The output and capacity of the hybrid ESS that can meet the demand of the fluctuation rate confidence level are calculated. The sampling time period is from $6 \mathrm{am}$ to $6 \mathrm{pm}$, and the sampling step is 6 seconds. The maximum and minimum output of $\mathrm{PV}$ is $466.25 \mathrm{~kW}$ and $1.25 \mathrm{~kW}$. The fluctuation rate demand is less than $10 \%$ per minute.

The sample output curve is shown is Fig. 2.

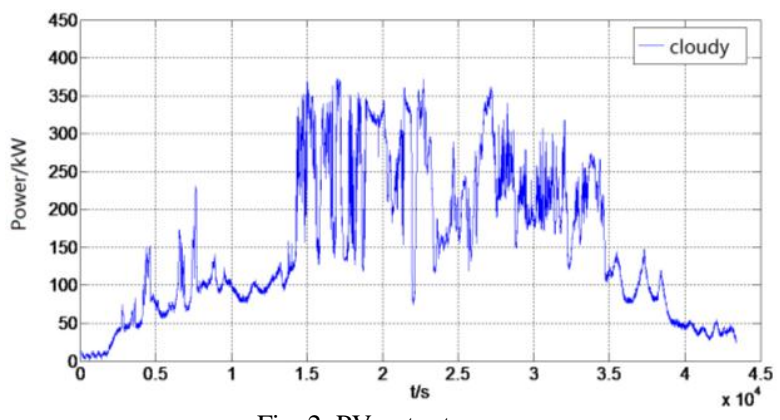

Fig. 2. PV output curve.

Apply spectral analysis, we can have the frequency domain curve as shown in Fig. 3.

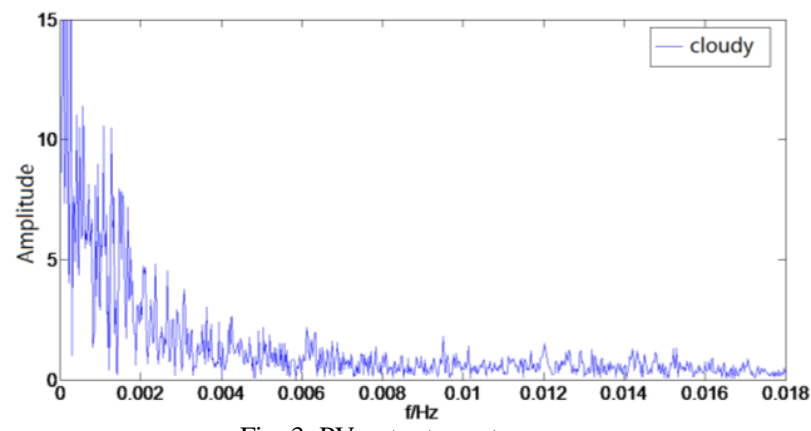

Fig. 3. PV output spectrogram.

From Fig. 3 we can see that, the high frequency fluctuation of PV output located between $6 \times 10-5 \sim 18 \times 10-3 \mathrm{~Hz}$, the corresponding filtering time constant is $1 \mathrm{~min}-277 \mathrm{~min}$.

From frequency-trying method, the fluctuation rate corresponding to different filer time constants are shown in Table I. 
TABLE I: FluCtUATING RATE CORRESPONDING TO DIFFERENT FILTER TIME CONSTANT

\begin{tabular}{cccccccc}
\hline \hline$T_{1}(\min )$ & 1 & 1.5 & 2 & 2.5 & 3 & 3.5 & 4 \\
\hline $\mathrm{F}_{\mathrm{tmax}}(\%)$ & 28.7 & 25.3 & 22.4 & 18.6 & 15.7 & 12.1 & 10 \\
$\mathrm{P} \%$ & 95.3 & 96.2 & 97.4 & 98.1 & 98.9 & 99.5 & 100 \\
\hline \hline
\end{tabular}

It can be seen that, when the confidence level are $100 \%$ and $95 \%$, the corresponding first-order filtering time constant $T_{1}$ are $4 \mathrm{~min}$ and $1 \mathrm{~min}$. From the charging and discharging characteristics of super capacitor, and considering the sampling cycle of PV, we choose $12 \mathrm{~s}, 20$ s and 30 s as the second-order filtering time constants. Simulation results are shown in Table II.

TABLE II: OUTPUT AND CAPACITY CONFIGURATION AND OF HYBRID ENERGy STORAGE SySTEM CORRESPONDING TO DIFFERENT FILTER TIME

\begin{tabular}{|c|c|c|c|c|c|c|c|}
\hline \multicolumn{8}{|c|}{ CONSTANT } \\
\hline $\begin{array}{l}T_{1} \\
(\min )\end{array}$ & $\begin{array}{c}P_{E N} \\
(\mathrm{~kW})\end{array}$ & $\begin{array}{l}E_{N} \\
(\mathrm{kWh})\end{array}$ & $\begin{array}{l}T_{2} \\
(\mathrm{~s})\end{array}$ & $\begin{array}{c}P_{\text {batN }} \\
(\mathrm{kW})\end{array}$ & $\begin{array}{l}E_{\text {batN }} \\
(\mathrm{kWh})\end{array}$ & $\begin{array}{c}P_{S C N} \\
(\mathrm{~kW}) \\
\end{array}$ & $\begin{array}{l}E_{S C N} \\
(\mathrm{kWh}) \\
\end{array}$ \\
\hline \multirow{3}{*}{1} & \multirow{3}{*}{217.6} & \multirow{3}{*}{11.96} & 12 & 186.1 & 12.5 & 178.8 & 1.37 \\
\hline & & & 20 & 184.1 & 12.6 & 181.4 & 1.67 \\
\hline & & & 30 & 180.6 & 12.7 & 182.9 & 1.75 \\
\hline \multirow{3}{*}{4} & \multirow{3}{*}{253.1} & \multirow{3}{*}{40.6} & 12 & 251.3 & 41.1 & 172.8 & 1.41 \\
\hline & & & 20 & 248.8 & 41.3 & 173.8 & 1.71 \\
\hline & & & 30 & 240.2 & 41.4 & 176.7 & 1.78 \\
\hline
\end{tabular}

For lack of space, only some of the Matlab simulation results are shown below. $\left(T_{1}=1 \mathrm{~min}, T_{2}=20 \mathrm{~s} ; T_{1}=4 \mathrm{~min}\right.$, $T_{2}=12 \mathrm{~s}$ )
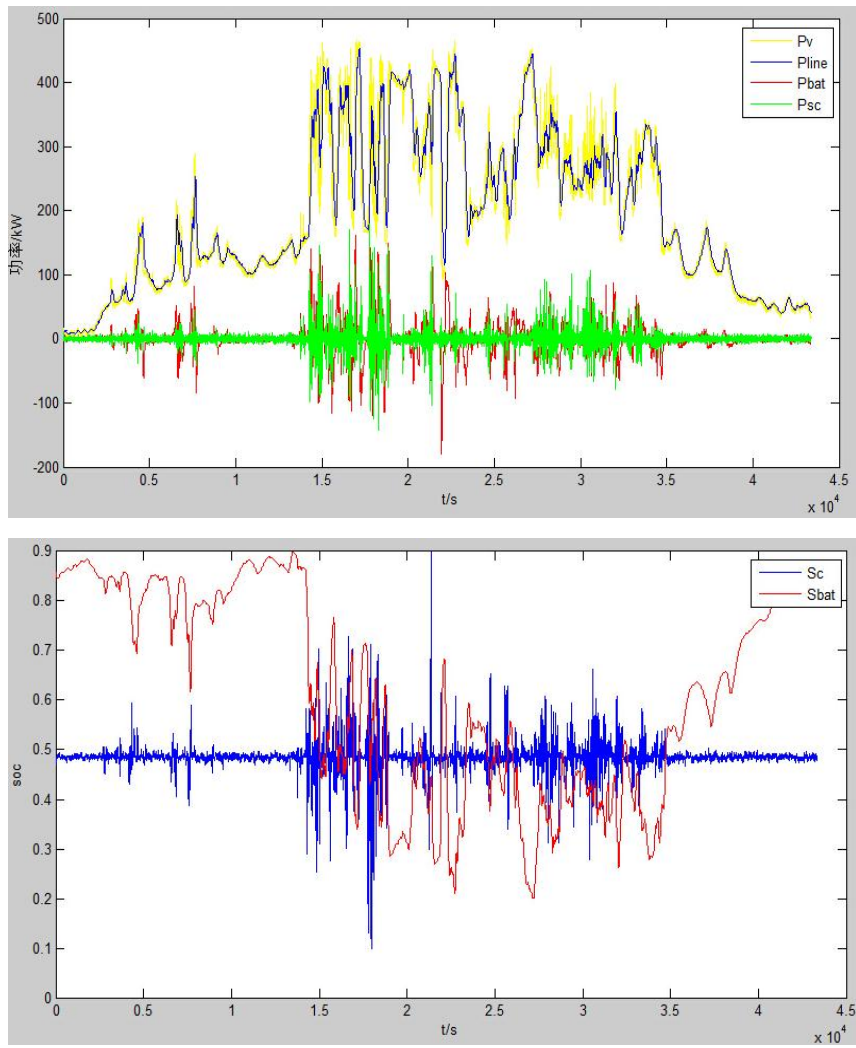

Fig. 4. Output of the hybrid generation system and the SOC operating curve of Li-battery, super capacitor.

The simulation curve has shown the real operating status of the hybrid ESS. The SOC of the battery varies from 0.9 to its initial state after a whole day of charging and discharging cycle. The SOC of the super capacitor is around 0.49 all the time, which is its initial state. From Table II, Fig. 4 and Fig. 5 we can see that, the larger the first-order filtering time constant is, the higher the confidence level is. Also the output curve of the hybrid ESS is more smooth, and the capacity of the ESS that need to installed is larger. If the first-order filtering time constant stays the same, the power of super capacitor increases slightly with the increase of the second-order filtering time constant. The output and capacity of the Li-battery doesn't change much, but the charging and discharging time become less. In real application, we can choose appropriate second-order filtering time constants according to the objective of the fluctuation rate and the performance of the battery.
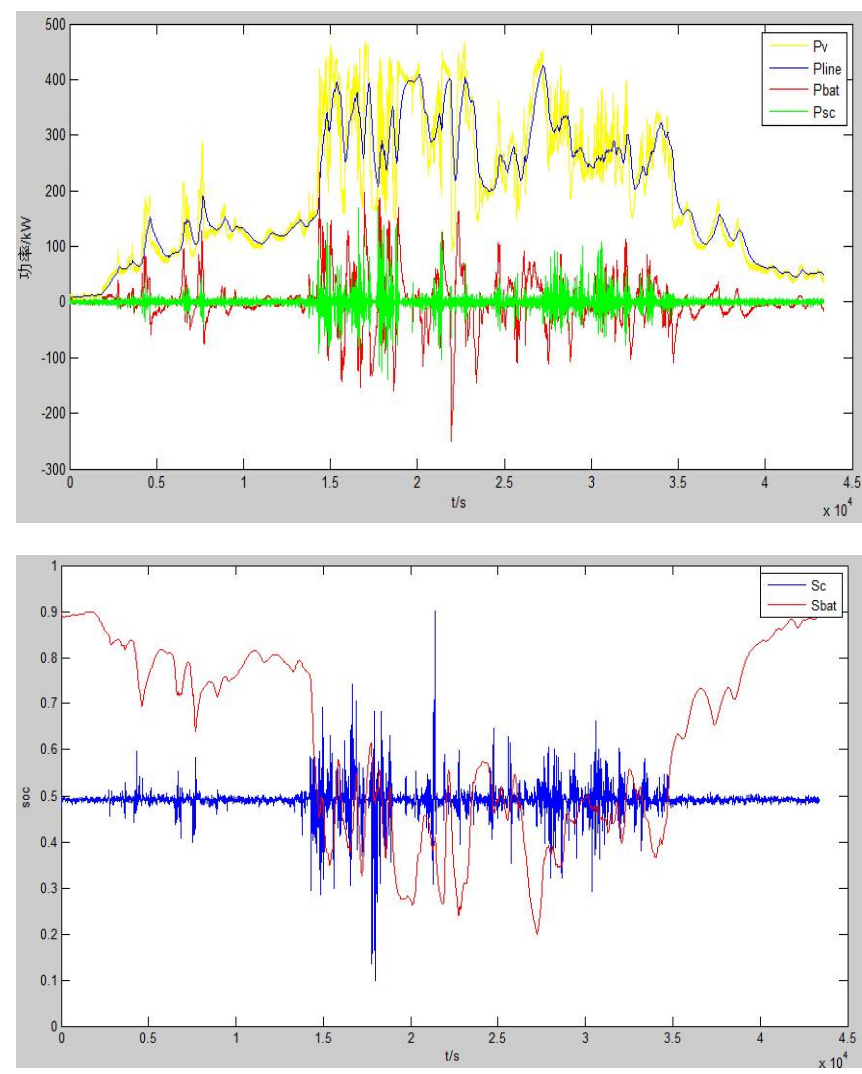

Fig. 5. Output of the hybrid generation system and the SOC operating curve of Li-battery, super capacitor.

In conclusion, the fluctuation rate of the power of the connecting line is restrained after adding hybrid ESS. The SOC curve of the Li-battery is smoother and went to its initial state after a period cycle. The super capacitor operated around its initial state, and its SOC curve is sharper. The rapid charging and discharging property of the super capacitor is fully used and the operating status of the Li-battery is optimized.

\section{CONCLUSION}

This paper proposed a capacity and output configuration method for hybrid EES considering the confidence level of $\mathrm{PV}$ output fluctuation rate. The fluctuation rate confidence level, power fluctuation rate constraint, energy balance constraint, charging and discharging efficiency of ESS and SOC is considered to get the output of the hybrid ESS that can meet the fluctuation rate demand. The output and capacity of the components of the hybrid ESS are also obtained. Simulation is performed in a $10 \mathrm{kV}$ distribution network 
system. The rated power and capacity of the Li-battery and super capacitor considering different fluctuation rate confidence level are obtained. The proposed method has a guiding significance for the application of distributed power source in distribution network.

\section{REFERENCES}

[1] C. H. Liang and X. Z. Duan, "Distributed generation and its impact on power system," Automation of Electric Power Systems, vol. 25, no. 12 pp. 53-56, 2001.

[2] Z. X. Lu, C. X. Wang, Y. Min et al., "Overview on microgrid research," Automation of Electric Power Systems, vol. 31, no. 19, pp 100-107, 2007.

[3] G. J. Zhang, X. S. Tang, and Z. P. Qi, "Energy storage system of super-capacitors and batteries in a microgrid," Automation of Electric Power Systems, vol. 34, no. 12, pp. 85-89, 2010.

[4] R. A. Dougal et al., "Power and life extension of battery-unltracapacitor hybrids," IEEE Trans on Components and Packaging Technologies, vol. 25, no. 1, pp. 120-131, 2002.

[5] H. B. Wang, X. Yang, and M. X. Zhang, "A control strategy of hybrid energy storage system capable of suppressing output fluctuation of photovoltaic generation system," Power System Technology, vol. 37, no. 9, pp. 52-58, 2013.

[6] L. Zhou, Y. Huang, K. Guo et al., "A survey of energy storage technology for micro-grid," Power System Protection and Control, vol. 39, no. 7, pp. 147-152, 2011.

[7] H. F. Wang, J. Cao, J. J. Qiu et al., "An active power compensation model for grid-connected distributed generation system," Automation of Electric Power Systems, vol. 33, no. 8, pp. 94-98, 2009.

[8] X. J. Han, C. Chen, T. Ji et al., "Capacity optimal modeling of hybrid energy storage systems considering battery life," Proceedings of CSEE vol. 33, no. 34, pp. 91-97, 2013.
[9] S. X. Xie, L. Yang, and L. Li, "Chance constrained pro-gramming based optimal configuration method of hybrid energy storage system," Power System Technology, vol. 36, no. 5, pp. 79-84, 2012.

[10] Y. You, D. Liu, and Q. Zhong, "Multi-objective optimal placement of energy storage system in an active distribution network," Automation of Electric Power Systems, vol. 38, no. 18, pp. 46-52, 2014.

[11] X. S. Tang, "Research on energy management and stability of distributed generation system with EDLC as energy storage," Dissertation, Chinese Academy of Sciences, 2006.

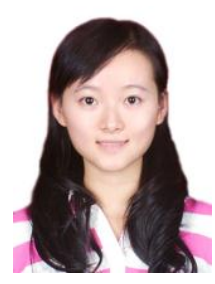

Jin Zong was born in Jiangxi province, China, in 1988 She received the master's degree in electrical engineering from North China Electric Power University in 2012. Currently, she is with State Grid Jibei Electric Power Co., Ltd Research Institute. Her main research interest is power system operation and distributed solar technology.

Kai Bai was born in Inner Mongolia province, China, in 1971. She received the master's degree in electrical engineering from Tsinghua University in 1997. Currently, she is with the State Grid Jibei Electric Power Co., Ltd Research Institute. Her main research interest is new energy technologies.

Bo Yuan was born in Henan province, China, in 1987. He received the B.S degrees in mathematics from Nankai University in 2009, and in electrical engineering from North China Electric Power University in 2014, respectively. Currently, he is with State Grid Energy Research Institute. His main research interest is power system reliability analysis and operation control.

Zhi Li was born in Jiangsu province, China, in 1987. He received the master's degree in electrical engineering from Heriotwatt University in 2011. Currently, he is with State Grid Jibei Electric Power Co., Ltd Research Institute. His main research interest is solar power technology. 